\title{
PET/CT Attenuation Correction: Breathing
}

\section{Lessons}

$\mathbf{T}$ hree articles in this issue of The Journal of Nuclear Medicine (1-3), and a concurrent one in the Journal of Nuclear Cardiology (4), remind us that the switch from stand-alone PET to PET/CT does involve some trade-offs. In particular, the effects of respiratory motion on the accuracy of PET data can be greatly exacerbated when CT-based attenuation correction is used. It seems worthwhile to think about some of the reasons why this is so before evaluating the various approaches to correct the problem that are put forth in this issue.

Combined PET/CT scanners have become the de facto standard for PET.

See pages 758,794 , and 811

There are significant diagnostic benefits from combining high-resolution anatomic images with functional PET data, and there seem to be great operational benefits as well-in particular, reduced imaging time. Part of the reduction in imaging time is a result of using the CT data (scaled to $511 \mathrm{keV}$ ) to perform attenuation correction. Until the advent of PET/CT scanners, attenuation correction was performed most often with a source of coincident $511-\mathrm{keV} \gamma$-rays (e.g., ${ }^{68} \mathrm{Ge}$ ) that rotated around the patient. By comparing the number of photons reaching the PET detectors with and without

Received Nov. 22, 2006; revision accepted Dec. 15, 2006.

For correspondence or reprints contact: Stephen L. Bacharach, PhD, Department of Radiology, University of California-San Francisco, 185 Berry

St., Suite 350, San Francisco, CA 94107.

E-mail: steve-bacharach@nih.gov

COPYRIGHT (c) 2007 by the Society of Nuclear

Medicine, Inc.

DOI: 10.2967/jnumed.106.037499 the patient, one can accurately compute the attenuation produced by the patient. This procedure is usually called a transmission scan or an attenuation scan. The procedure is exactly like a CT scan, but with the rotating $\mathrm{x}$-ray tube replaced by a ${ }^{68} \mathrm{Ge}$ source. Unfortunately, even when multiple ${ }^{68} \mathrm{Ge}$ sources were installed in the PET scanner, the process took 3-10 min for each bed position. The reason is that the number of photons emitted by even the strongest ${ }^{68} \mathrm{Ge}$ source that the PET machine could handle (typically around $370 \mathrm{MBq}$ ) still limited the total counting rate, requiring several minutes to acquire adequate statistics. This additional 310 min per bed position did not greatly affect single-bed-position cardiac imaging but dramatically affected the time required for oncologic applications (typically 5-7 bed positions). Combining a CT scanner with the PET scanner offered the chance to replace the ${ }^{68} \mathrm{Ge}$ rotating rod source with a CT $\mathrm{x}$-ray source to perform the attenuation correction. Because $\mathrm{x}$-ray sources produce huge numbers of photons, adequate statistics are obtained within seconds even at low tube currents (5). Current-generation CT scanners have speeds of half a second per rotation and can acquire a spiral scan of each $15-\mathrm{cm}$ bed position in only a few seconds. Even at low tube currents, however, the radiation exposure from CT can be between 1 and 2 orders of magnitude larger than the small dose from the ${ }^{68} \mathrm{Ge}$ source (tens of millirads at most). Still, CT-based attenuation correction seemed an important improvement in attenuation correction. Even in the presence of contrast media, CT-based attenuation coefficients have been shown to be quite accurate (unless very high contrast concentrations were present).

The CT-based method of attenuation correction was quickly and widely adopted for clinical use. Most clinical PET/CT machines sold today no longer come equipped with the ${ }^{68} \mathrm{Ge}$ source and rely solely on CT-based attenuation correction. Unfortunately, a problem was soon noted in certain oncologic applications. Erroneous values of ${ }^{18} \mathrm{~F}$-FDG uptake were often found near the dome of the liver and in lung tumors. The same feature that made CT attenuation correction so attractive-its high speed-also turned out to be the cause of these artifacts. It had been long known $(6,7)$ that misalignment between the PET emission data and the ${ }^{68} \mathrm{Ge}$ transmission data could produce incorrect values of ${ }^{18} \mathrm{~F}$ FDG uptake. When ${ }^{68} \mathrm{Ge}$ sources were used, this misalignment usually was caused by motion of the patient between the transmission scan and the subsequent emission scan. The artifact produced by such motion could be quite severe and occurred primarily at borders between low-attenuation tissue (e.g., lung) and soft tissue. Imagine, for example, a tumor near the dome of the liver, seen on slice 20 of the emission data. The photons emitted by this tumor might have to traverse many centimeters of liver, resulting in significant attenuation. Slice 20 of the transmission scan would show the presence of this liver tissue and make the appropriate (large) attenuation correction, boosting the measured number of photons, yielding the correct value of tumor uptake. If, however, the patient should move during the course of either scan, slice 20 of the transmission scan would be in a location different from slice 20 of the emission scan. Slice 20 of the transmission scan might, for 
example, show no liver, only lung. In this case, the transmission correction would be far too low-the machine would "think" that the photons from the tumor in slice 20 had passed only through lung, not liver, tissue. The tumor intensity would no longer be boosted by the attenuation correction and, so, would be greatly underestimated.

In the days of ${ }^{68} \mathrm{Ge}$ transmission sources, such misalignment could be caused only by motion of the patient between the 2 scans. CT-based attenuation correction should reduce this potential patient motion because of the shorter time the patient is on the table. However, CT attenuation correction has introduced a new source of motion: respiratory motion. Respiration causes many organs and tissues to move quite substantial distances, especially near the diaphragm $(1-4,8,9)$. The dome of the liver, the kidneys, and other tissues can move many centimeters over the course of a normal respiratory cycle. This motion was not a problem for ${ }^{68} \mathrm{Ge}$ attenuation correction. Respiratory motion blurred the emission data, because many respiratory cycles were averaged during the many minutes of emission acquisition. The ${ }^{68} \mathrm{Ge}$ transmission data were equally blurred, again because data were acquired over several minutes. Unless the patient moved, the blurred transmission data exactly matched the blurred emission data, provided the patient was breathing in a similar manner for both scans. The advent of CTbased attenuation correction brought about a change. The emission data still took several minutes to acquire and so remained blurred by the respiratory cycle. The CT, however, took only a short time-often less than a second for each slice. Thus, the CT scan of that slice was frozen in time in whatever stage of the respiratory cycle the patient happened to be. People tried to compensate by asking patients to breathe only very shallowly during $\mathrm{CT}$, or even to hold their breath at end expiration (the part of the cycle that dominates a normal breathing pattern) (10). No matter which part of the cycle was captured by the CT, however, it would never be the same as would be achieved by the averaging process inherent in the emission and ${ }^{68} \mathrm{Ge}$ transmission measurements, potentially producing significant errors in ${ }^{18} \mathrm{~F}$-FDG uptake. For the heart, the situation is potentially worse. Much of the myocardium has a boundary with the lung and is only about $10 \mathrm{~mm}$ thick. A few millimeters of respiratory motion could easily make the myocardium in the emission data appear in the lung field of the CT data. Because only part of the myocardium borders the lung, only part of the myocardium would have this problem, producing nonuniformities in the cardiac images. Such nonuniformities could easily be mistaken for perfusion defects. Despite some contradictory findings in the past (11), several authors, including those in this issue of The Journal of Nuclear Medicine, have pointed out that the problem can be significant, especially for cardiology (4).

How can one correct this problem? One solution would be to average the CT data over many respiratory cycles $(12,13)$, in the same way that the rotating rod source and emission data are an average over many respiratory cycles. There are several approaches to this averaging, some of which are outlined in this issue. The most obvious would be simply to slow the rotational speed of the $\mathrm{x}$-ray tube enough for a single revolution to encompass a complete respiratory cycle. This approach, however, could introduce severe artifacts into the CT data caused by each projection's "seeing" the body at a different phase of the respiratory cycle. The resulting inconsistent projections are known to produce artifacts in the reconstructed image. The rotating rod overcomes this difficulty because so many rotations are needed that each projection angle is the average of the data from many respiratory cycles. Of course, if one could lower the beam current enough (often not possible on current CT scanners), one could do the same with the X-ray tube of the CT scanner. This method would prevent one from performing a helical scan, however, and might begin to take as long to perform as would the use of the rotating rod. Another approach would be to obtain a cine CT scan, which could span one or more respiratory cycles (as in some of the current papers). This type of scan increases the attenuation time required, but not excessively so. Radiation exposure is an issue but can be controlled with lower beam currents (5). Again, synchronization of the CT with the respiratory cycle would seem to be a problem, but in one of the papers in this issue, this problem is shown not to matter much. Another similar approach would be to gate the CT to the respiratory cycle $(14,15)$. One could then average the respiratory gated data to match the ungated PET data. Alternatively, one could respiratory-gate both the PET emission data and the CT data. Then, the attenuation correction data at each phase of the respiratory cycle would be matched to the emission data at the corresponding phase. Both these approaches have been explored in the current papers. The double-gating technique takes a heroic amount of data processing but would potentially have the advantage of also eliminating the spatial blurring of the emission data caused by respiratory motion. Statistics would be quite limited, but one could in theory realign each gate of the attenuation-corrected gated PET data and sum them. In fact, one of the current papers (2) looks at the feasibility of using "center-of-mass (activity)" measurements to perform similar alignments.

The set of 4 new papers published this month in The Journal of Nuclear Medicine and in a recent issue of Journal of Nuclear Cardiology (4) have undoubtedly been strongly influenced by the considerable work done previously by others in this area. The papers serve both to underscore the potential magnitude of the problem and to suggest and validate clinically feasible solutions. Yes, these solutions may take some extra effort, but several could easily be incorporated into clinical practice (with help from the manufacturers). We really cannot afford to ignore this problem. Only by addressing the effects of respiratory 
motion can we hope to generate quantitatively accurate PET/CT images of tumors or the myocardium.

\section{Stephen L. Bacharach University of California} San Francisco, California

\section{REFERENCES}

1. Alessio AM, Kohlmyer S, Branch K, Chen G, Caldwell J, Kinahan P. Cine CT for attenuation correction in cardiac PET/CT. J Nucl Med. 2007;48:794-801.

2. Bundschuh RA, Martinez-Moeller A, Essler M, et al. Postacquisition detection of tumor motion in the lung and upper abdomen using list-mode PET data: a feasibility study. J Nucl Med. 2007;48:758-763.

3. Cook RAH, Carnes G, Lee T-Y, Wells RG. Respiration-averaged $\mathrm{CT}$ for attenuation correction in canine cardiac PET/CT. J Nucl Med. 2007;48:811-818.
4. Le Meunier L, Maass-Moreno R, Carrasquillo JA, Dieckmann W, Bacharach SL. PET/CT imaging: effect of respiratory motion on apparent myocardial uptake. J Nucl Cardiol. 2006;13:821-830.

5. Kamel E, Hany TF, Burger C, et al. CT vs Ge-68 attenuation correction in a combined PET/CT system: evaluation of the effect of lowering the CT tube current. Eur J Nucl Med Mol Imaging. 2002;29:346-350.

6. McCord ME, Bacharach SL, Bonow RO, Dilsizian V, Cuocolo A, Freedman N. Misalignment between PET transmission and emission scans: its effect on myocardial imaging. J Nucl Med. 1992; 33:1209-1214.

7. Ter-Pogossian M, Bergmann S, Sobel B. Influence of cardiac and respiratory motion on tomographic reconstructions of the heart: implications for quantitative nuclear cardiology. J Comput Assist Tomogr. 1982;6:1148-1155.

8. Goerres GW, Kamel E, Heidelberg TNH, et al. PET-CT image co-registration in the thorax: influence of respiration. Eur J Nucl Med Mol Imaging. 2002;29:351-360.
9. Huesman RH, Klein GJ, Reutter BW, et al. List mode data acquisition for retrospective respiratory-cardiac gated PET [abstract]. J Nucl Med. 1998;39(suppl):93P.

10. de Juan R, Seifert B, Berthold T, et al. Clinical evaluation of a breathing protocol for PET/CT. Eur Radiol. 2004;14:1118-1123.

11. Koepfli P, Hany TF, Wyss CA, et al. CT attenuation correction for myocardial perfusion quantification using a PET/CT hybrid scanner. J Nucl Med. 2004;45:537-542.

12. Pan T, Mawlawi O, Nehmeh SA, et al. Attenuation correction of PET images with respirationaveraged CT images in PET/CT. J Nucl Med. 2005; 46:1481-1487.

13. Pan T, Mawlawi O, Luo D, et al. Attenuation correction of PET cardiac data with low-dose average CT in PET/CT. Med Phys. 2006;33:3931-3938.

14. Nehmeh SA, Erdi YE, Pan T, et al. Fourdimensional (4D) PET/CT imaging of the thorax. Med Phys. 2004;31:3179-3186.

15. Pan T, Lee TY, Rietzel E, et al. 4D-CT imaging of a volume influenced by respiratory motion on multi-slice CT. Med Phys. 2004;31:333-340. 